\title{
Receding Horizon Control Applied to Optimal Mine Planning
}

\author{
Graham C. Goodwin $^{\text {a }}$ María M. Seron ${ }^{\text {a }}$ Richard H. Middleton ${ }^{\text {a }}$ \\ Meimei Zhang ${ }^{\mathrm{a}, \mathrm{b}} \quad$ Bryan F. Hennessy ${ }^{\mathrm{a}} \quad$ Peter M. Stone $^{\mathrm{b}} \quad$ Merab Menabde $^{\mathrm{b}}$ \\ ${ }^{a}$ Centre for Complex Dynamic Systems and Control, School of Electrical Engineering and Computer Science, The University \\ of Newcastle, Callaghan 2308, NSW, Australia \\ ${ }^{\mathrm{b}}$ BHP Billiton, GPO Box 86A, Melbourne VIC 3001, Australia
}

\begin{abstract}
In this paper we show that the problem of optimal mine planning can be cast in the framework of receding horizon control. Traditional formulations of this problem have cast it in the framework of mixed integer linear programming. In this paper, we present an alternative formulation of the mine planning problem using the "language" of control engineering. We show that this alternative formulation gives rise to new insights which have the potential to lead to improved computational procedures. The advantages are illustrated by an example incorporating many practical features of an actual mine planning problem.
\end{abstract}

\section{Introduction}

Mining companies throughout the world have recognised that considerable economic gains are achievable through careful planning of mining operations. Indeed, there exists a substantial, and growing, literature on this topic see, for example, $[10,7,12,13,16,17,15,14,25,5,6,9]$. Also, several commercial software packages are available which can be used for mine planning — see [1-4].

Many algorithms have been used for the purpose of mine planning. Typical procedures, e.g., [24] formulate the problem as a mixed integer linear programme (MILP). A variety of solution strategies have been used including the commercially available software CPLEX, branch and cut methods [10], genetic algorithms [7,12-17], simulated annealing [25], etc.

A complete study of mine planning involves a myriad of diverse issues and constraints, including: plant capacity constraints; geotechnical constraints on mine wall slopes; multiple processing plants with variable capacities, process rates and operating costs; mine access constraints (i.e., the need for sufficient flat areas and access roads for mining equipment); multiple material stockpiles; variable material price; blending and mixing issues to attain a target material grade; uncertainty in ore-body composition; evaluation of "real options" (e.g., the ability to

\footnotetext{
1 Corresponding author. Maria.Seron@newcastle.edu.au
}

purchase additional equipment during the mine's operation in response to a higher than expected market price). Necessarily, therefore, with the complexity and size of such optimisation problems, the problem is subdivided, and a variety of simplifying assumptions are made to give a more tractable set of subproblems.

In the current paper we propose an alternative formulation of the mine planning problem under plant capacity constraints and geotechnical constraints on mine wall slopes. Specifically, we define the mine state as a collection of pit depths at a certain number of surface locations and we represent the evolution of this state via a dynamic model that uses mining action as control input. We then use receding horizon optimal control $[11,19,8,23,18]$ to solve the mine planning problem under constraints. This leads to several advantages, some of which are explained in the main body of the paper.

The layout of the remainder of the paper is as follows: In Section 2 we present the problem formulation. In Section 3, we explain how receding horizon control can be applied to this problem. In Section 4 we describe preliminary results for a realistic example. In Section 5 we discuss computational issues associated with the receding horizon solution and make comparisons with a traditional fixed horizon approach. Finally, in Section 6 we draw conclusions. 


\section{Problem Formulation}

To give a "feel" for what a mine actually looks like, we show in Figure 1 a typical final (ultimate) pit after mining operations have been completed.

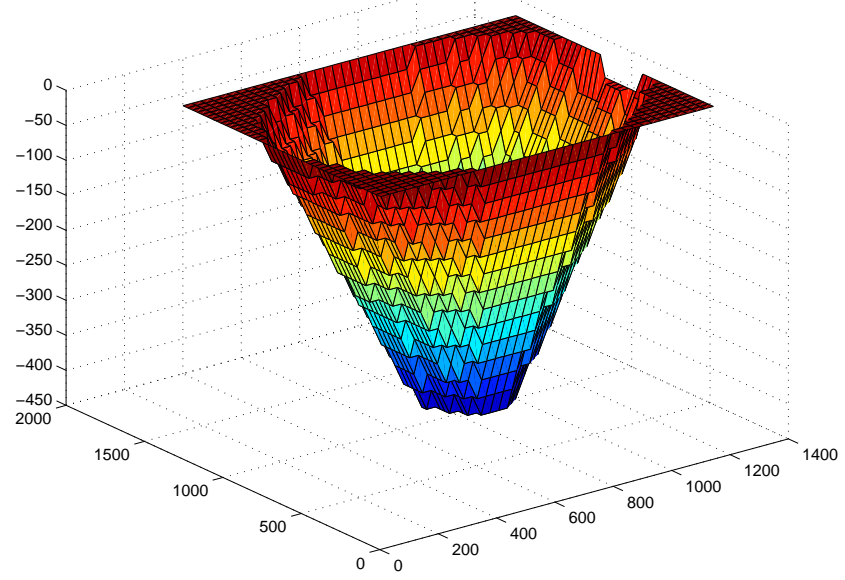

Fig. 1. Ultimate pit

We represent the mine by the three dimensional diagram shown in Figure 2. The top of the box represents the mine surface, which we divide into $N_{y} \times N_{z}$ rectangles.

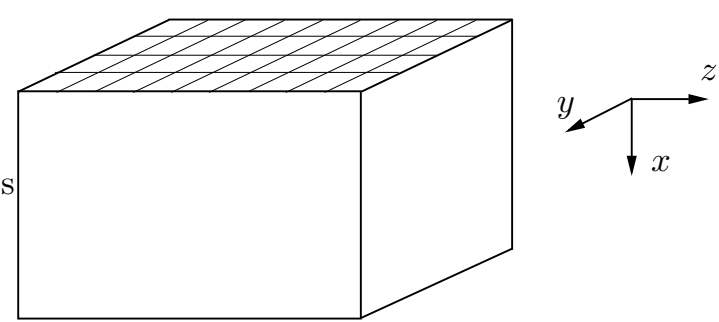

Fig. 2. Representation of open pit mine

\subsection{Inputs}

We denote by $u_{i j}(k)$ the action to mine (or not) at time $k$ in the area $i j, i \in\left\{1, \ldots, N_{y}\right\}, j \in\left\{1, \ldots, N_{z}\right\}$. We thus think of $u_{i j}(k)$ as an $N_{y} \times N_{z}$ input vector.

\subsection{States}

We denote by $x_{i j}(k)$ the mine depth (or profile) at location $i j$ at time $k$. A state model for the system can then be written as

$$
\begin{aligned}
x_{i j}(k+1) & =x_{i j}(k)+c_{1} u_{i j}(k), \quad k \geq 1, \\
x_{i j}(1) & =0, \quad i=1, \ldots, N_{y}, \quad j=1, \ldots, N_{z},
\end{aligned}
$$

where $c_{1}$ is a constant that reflects the effect of one unit of mining action.

\subsection{Input Constraints}

We note that $u_{i j}(k)$ is drawn from a finite alphabet, that is, $u_{i j}(k)$ can be either 0 (absence of mining at location $i j$ at time $k$ ) or 1 (mining at location $i j$ at time $k$ ). Also, only a certain number, determined by the mining capacity, of $u_{i j}(k)$ can be nonzero at any $k$.

\subsection{State Constraints}

Mining strategies need to satisfy a number of constraints on the order that material can be mined - see $[10,7,12,13,16,17,15,14,25,5,6,9,24]$. In the usual MILP formulation these constraints are typically included as a set of precedence constraints. In the alternative "control engineering" formulation described here, the constraints are readily incorporated. For example, since $u_{i j}(k)$ is nonnegative then (1) immediately ensures that the mine depth cannot decrease and that we cannot "undermine" a given location. Also, "slope" constraints on the mining depth are readily incorporated by using state constraints of the form

$$
\begin{aligned}
& \qquad\left|x_{\ell n}(k)-x_{i j}(k)\right| \leq c_{2} \quad \forall k \geq 1, \\
& \text { for } \\
& \quad|\ell-i|=1 \\
& \quad|n-j|=1 .
\end{aligned}
$$

Note that the constraints represented in (2) are linear inequalities, and therefore are convex in $x_{i j}(k)$.

\subsection{Other Constraints}

Processing plant constraints can also be modelled by introducing functions to model ore content. The details of such models will depend on whether or not stockpiling is considered as well. The methodology described here can be extended to these more complex scenarios. However, for simplicity we will restrict ourselves to the special case outlined above.

\subsection{The Value of Ore and Discounted Cost}

We assume that we know the value of the body of ore at location $i j$ at depth $x_{i j}$. This is typically obtained by preliminary drilling work. We denote the associated value function as $V_{i j}\left(x_{i j}\right)$. We also introduce time discounting (to yield net present value) through a time function $d_{k}$.

The total return from a given mining strategy will then take the form

$$
J=\sum_{k=1}^{T} \sum_{i=1}^{N_{y}} \sum_{j=1}^{N_{z}} d_{k} V_{i j}\left(x_{i j}(k)\right) u_{i j}(k) .
$$


Note that $T$ represents the planning horizon. Also, we multiply by $u_{i j}(k)$ in (3) since the value in the ore is only liberated when it is mined.

In the formulation described above, there are $N_{y} N_{z}$ continuous state variables. However, since the input takes discrete values, this implies that the continuity of the states variables is fictitious. Indeed, the states can only take values in a discrete set. Of course, it would be possible to approximate the input constraints by relaxing the finite alphabet nature of the input and instead use variables that take continuous values. Unfortunately, this does not appear to offer major advantages since an overriding issue is the nonconvexity of the cost function (3). This means that the problem is fundamentally complex from a computational viewpoint. Thus one has to use various techniques such as aggregation to reduce complexity. In this regard, the receding horizon idea described next is a helpful tool.

\section{Application of Receding Horizon Control to Mine Planning}

Receding horizon optimisation has become a very successful strategy in real time control problems $[11,19,8,23,18]$. The basic idea is that one solves a fixed horizon optimisation problem to compute a sequence of predicted inputs over some prediction horizon (say $T$ time steps) but one only implements (or stores) the first step. Then time is advanced one step and the process is repeated. This strategy has been enormously successful in process control applications [20].

Note that various simplifications are known to work well in the process control case $[11,19,8,23,18]$. For example,

(1) The input is usually deemed to be constant after some period. The rationale for this is that the fine detail of controls in the distant future have minimal impact on the current optimal action.

(2) Often constraints can be relaxed beyond some (small) prediction horizon. Again, the rationale is that fine detail in the distant future has minimal impact on the current optimal action.

Although receding horizon optimisation was originally developed to facilitate real-time issues, the idea has also been used as a way or simplifying computational demand when there is no real-time constraint, see [21]. In the sequel, we outline how points (1) and (2) above can be captured in the context of the mine planning problem.

\subsection{Time Quantisation}

For the mine planning problem, we propose to use nonuniform discretisation of time (with corresponding changes in the mining capacity constraint). This aggregates future mining operations based on the hypothesis that actions taken in the far future do not have a significant impact on the best step to take next. Figure 3 shows a possible receding horizon solution strategy using geometrically quantised time for a planning horizon $T=15$ years. Each plot represents a sequence of predicted actions of increasing width (corresponding to the nonuniform time quantisation) obtained as the solution to a fixed horizon optimisation problem starting at years 1, 2 and 3, respectively. The shaded areas indicate the mining actions for years 1, 2 and 3, which are computed as the first step of the corresponding sequences. Note that only the shaded areas form part of the final solution. Thus, although nonuniform time quantisation is used in deriving the solution, only the first steps, of smallest width, are actually implemented. Hence, the final mining strategy is actually finely and uniformly quantised in time. The strategy also implies a constraint relaxation in the future since the state constraints (of the type (2)) would only be required to be satisfied at the end of each time quantised step. An important advantage of nonuniform time quantisation is complexity reduction. Indeed, for a planning horizon $T$ and $N_{s}$ surface locations, the complexity of the optimisation problem with uniform time quantisation $[1,2, \ldots, T]$ is $N_{s}^{T}$ whereas using nonuniform time quantisation with, say, 4 intervals, the complexity is $T N_{s}^{4}$. A specific computational example is given in Section 5 .

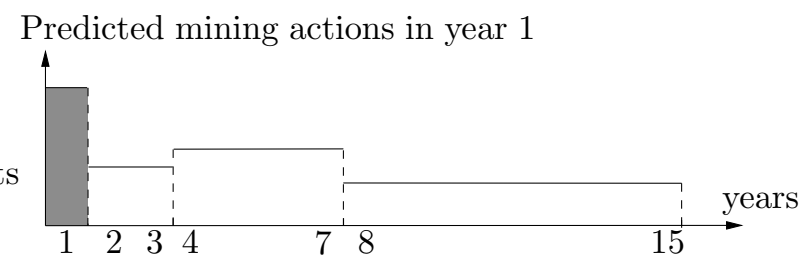

Predicted mining actions in year 2

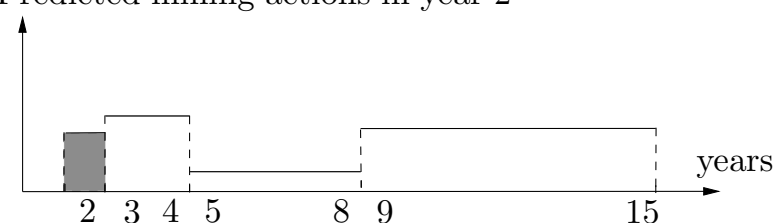

Predicted mining actions in year 3

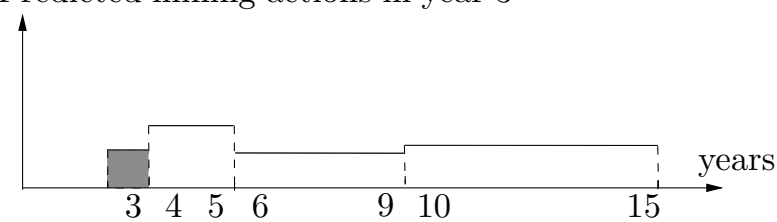

Fig. 3. Geometrically quantised time in receding horizon optimisation. (Only the shaded inputs are applied.)

In this context we note that the discount factor $d_{k}$ used in (3) to achieve net present value is necessarily quantised due to the discretisation of time. Nonuniform time quantisation has an advantage in this regard since it ensures that the errors induced in the quantisation of the discount factor are minimised. This is clarified in the following lemma. 
Lemma 1 Let $d(t)$ denote an exponentially decaying discount factor where

$$
d(t)=e^{-a t} \quad \text { for } \quad t \in[0, T] .
$$

Then the sequence $t_{1}, \ldots, t_{N}$ increasing such that

$$
\left\{t_{1}, \ldots, t_{N}\right\}=\arg \min _{t_{i} \in[0, T]} \max _{i}\left[d\left(t_{i-1}\right)-d\left(t_{i}\right)\right]
$$

where $t_{0}=0, t_{N}=T$, satisfies

$$
t_{i}=-\frac{\ln (1-i \alpha)}{a}
$$

where

$$
\alpha=\frac{1-e^{-a T}}{N} .
$$

PROOF. It is readily seen by contradiction that the times $t_{1}, \ldots, t_{N}$ must yield equal steps in the discount factor. The step in descent factor is then as in (6) and (5) follows from (4).

As an illustration, let $a=0.05, T=20$ and $N=5$. Then the optimal quantisation times are $t_{0}=0, t_{1}=2.7$, $t_{2}=5.8, t_{3}=9.5, t_{4}=14$ and $t_{5}=20$.

\subsection{Constraint Relaxation}

Other strategies that may alleviate the computational burden include constraint relaxation.

One form of constraint relaxation follows from time quantisation as outlined in Section 3.1. Other possible relaxations are outlined below.

(1) To further reduce computational complexity it is possible to parameterise the input in terms of a set of basis functions. Careful choice of these basis functions can aid the satisfaction of constraints. Thus, instead of saying that the input belongs to a finite alphabet, we could constrain the input to be one of a small set of basis functions. By way of illustration, we show one possible basis function in Figure 4 . This basis function corresponds to digging a subdivision of the pit called phase or pushback.

(2) Based on the principle enunciated in Section 3.1, we conjecture that one need not be too precise about the control action in the distant future since this is ultimately "washed away" in the receding horizon solution. This suggests that one could aggregate basis functions in accordance with the nonuniform quantisation scale.

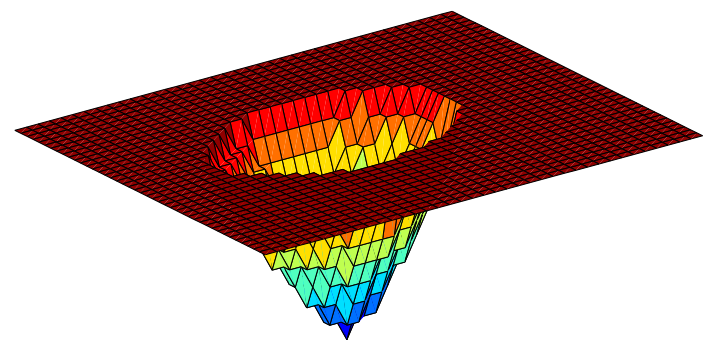

Fig. 4. Illustration of mining effort basis function.

\section{Example}

To illustrate the basic principles involved in applying receding horizon optimal control to mine planning, we choose a block model containing 9213 blocks corresponding to an actual mine. Each block has a known weight (average 60000 tons), dimensions $30 \times 30 \times 30 \mathrm{~m}^{3}$ and a pre-defined in-ground value. The mine surface (see Figure 2) has $N_{y} \times N_{z}=39 \times 43$ squares covering an area of $1170 \times 1290 \mathrm{~m}^{2}$. The mining input is measured in tons and the mining capacity is $4 \times 10^{7}$ tons per year. We also apply the constraint that no more than $2 \times 10^{7}$ tons of ore can be processed per year. We use the state space model (1), which has dimension 1347 for this example. The constant $c_{1}$ in (1) is taken to be (30m)/(60000tons). The objective is to obtain a yearly mining schedule based on a cost function of the form (3). The values $V_{i j}\left(x_{i j}\right)$ in (3) are interpolated from the in-ground values of the block model.

Other methods have been used to attack this mine planning problem (see e.g., $[10,24,25])$. Here we use some of the ideas described in Section 3, namely, nonuniform time quantisation and aggregation by means of basis functions, to give a simplified solution for this problem.

The following specifications were used:

(1) Planning horizon $T=13$ years.

(2) Discount function $d_{k}=(1 / 1.1)^{(k-1)}$ in the cost function (3).

(3) Time quantisation (as in Section 3.1) of 1, 1, 1, 10 years for the first step, followed by 1, 1, 1, 9 years, etc., that is, the horizon recedes 1 year in each of the 13 steps.

(4) Use of 7 input basis functions (one of which is shown in Figure 4) with variable quantisation; namely, in each of the 13 steps, the number of basis functions chosen was 3 for the 1-year long periods and 7 for the last period.

(5) A slope constraint of 1.

The optimisation was performed by evaluating all possibilities, which, on the one hand, is computationally tractable due to the strategy used and, on the other hand, guarantees that the solution is the global optimum for the problem formulation. The computa- 

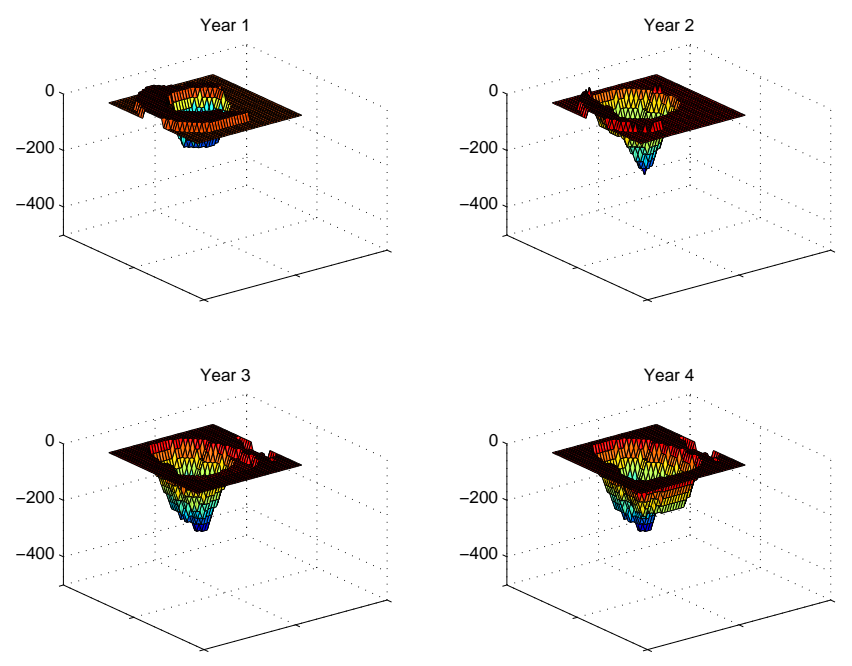

Fig. 5. Yearly schedule obtained via receding horizon control

tional complexity is $13 \times 3^{3} \times 7=2457$ (compare with $7^{13} \approx 10^{11}$ if we used 7 basis functions over 13 years with uniform - yearly - quantisation). The total return achieved is $\$ 740$ million (compare with $\$ 750$ million achieved using CPLEX with finer aggregation).

Figure 5 shows the yearly schedule over the first 4 years. These plots represent the actual states of the mine needed in yearly intervals. The final 13 th year is the ultimate pit. This was actually shown earlier in Figure 1.

\section{Some Computational Issues}

The connection between optimal mine planning and receding horizon control has led to results of practical relevance to the industrial partner involved in this project. Specifically, the idea of receding horizon control, including nonuniform time quantisation, has enabled significantly larger planning problems to be tackled than was previously possible. Also, note that the formulation of the problem presented here allows any appropriate solution method, including standard MILP solvers. This is further discussed in [22]. Specifically, the results presented below were obtained using the commercial package CPLEX. Seven tests were carried out for a practical mine planning problem, each test using a different number of units of aggregation. Care was taken during the aggregation process to ensure that conditions were equivalent. The total return is denoted here as NPV (net present value). All NPVs presented below are within $0.1 \%$ of their respective optimal value, save for the case where the number of aggregation units was 1632. In the latter case, the NPV is within $0.57 \%$ of the optimal value.

Figure 6 shows computation times, in seconds, as a function of aggregation units. Notice that there is an order of magnitude difference in computation time for aggregation units 1632 and the gap is growing. Interestingly, the receding horizon approach results in only a very small loss in NPV as shown in Figure 7. Moreover, at aggregation units of 1632, the receding horizon approach has resulted in a larger NPV due to computational difficulties with the fixed horizon solution.

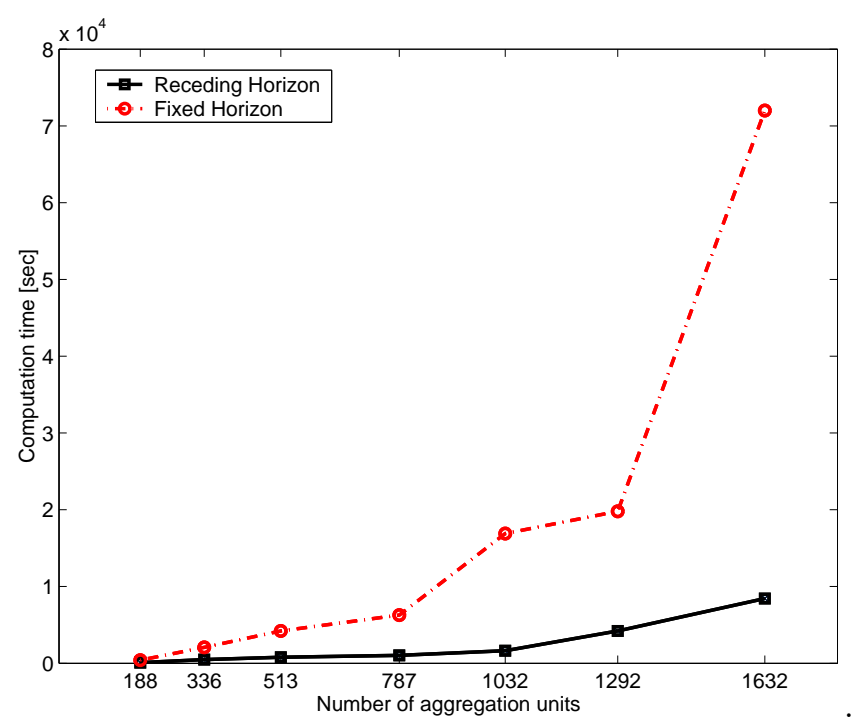

Fig. 6. Computation time of CPLEX to find the optimal solutions with receding horizon and fixed horizon

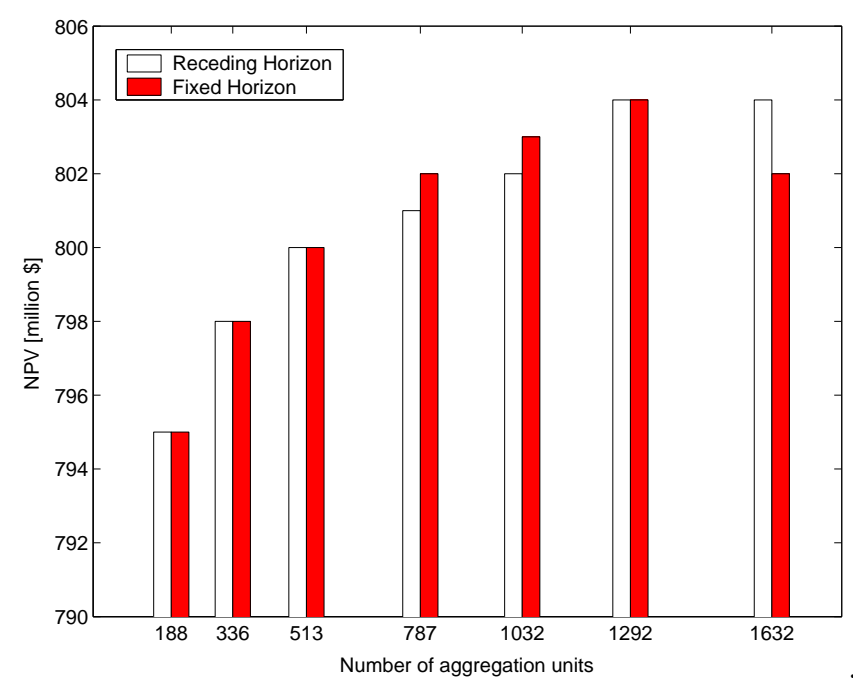

Fig. 7. Column plot of the NPV achieved by CPLEX with receding horizon and fixed horizon

\section{Conclusions}

This paper has described an alternative formulation of optimal mine planning as a receding horizon optimal control problem. It has been argued that this formulation yields advantages in terms of insight and computational complexity. Future work in this area will further exploit the control engineering formulation. For example, in recent work we have applied stochastic optimal 
control theory to problems of current interest in this industry sector including pricing flexibility.

\section{Acknowledgements}

We gratefully acknowledge the help of David Allingham of CDSC University of Newcastle. We also acknowledge BHP Billiton for providing the contextual basis for the problem, financial support and access to real data.

\section{References}

[1] Earthworks multimine scheduler. http://www.earthworks.com.au/.

[2] ECSI maximiser. http://www.ecsi.com.au/.

[3] MinMAX planner. http://www.minemax.com/.

[4] Whittle strategic mine planning. http://www.whittle.com.au/.

[5] P. B. Appiah, M. A. Rosenman, and J. R. Sturgul. Introduction to Pareto optimal mine planning. Journal of Mining and Geological Engineering, 8(4):348-356, 1990.

[6] P. B. Appiah and J. R. Sturgul. Pareto optimal stochastic mine planning. International Journal of Surface Mining, Reclamation and Environment, 4(4):181-195, 1990.

[7] M. Ataei and M. Osanloo. Using a combination of genetic algorithm and the grid search method to determine optimum cutoff grades of multiple metal deposits. International Journal of Surface Mining, Reclamation and Environment, 18(1):60-78, 2003.

[8] F. Borrelli. Constrained Optimal Control of Linear and Hybrid Systems. Springer, Heidelberg, 2003.

[9] M. Brazil, D. H. Lee, M. Van Leuven, J. H. Rubinstein, D. A. Thomas, and N. C. Wormald. Optimising declines in underground mines. Mining Technology: Trans. of the Institution of Mining and Metallurgy, Section A, 112:164$170,2003$.

[10] L. Caccetta and S. P. Hill. An application of branch and cut to open pit mine scheduling. Journal of Global Optimization, 27:349-365, 2003.

[11] E. F. Camacho and C. Bordons. Model Predictive Control. Advanced Textbooks in Control and Signal Processing. Springer, London, 1999.

[12] S. Clement and N. Vagenas. Use of genetic algorithms in a mining problem. International Journal of Surface Mining, Reclamation and Environment, 8:131-136, 1994.

[13] Paul J. Darwen. Genetic algorithms and risk assessment to maximize NPV with robust open-pit scheduling. In Fourth Biennial Conference on Strategic Mine Planning, pages 2934, Perth, Western Australia, 2001.

[14] B. Denby and D. Schofield. Open-pit design and scheduling by use of genetic algorithms. Transactions of the Inst. Min. Metall., Section A: Min. industry, 103:A21-A26, January April 19941994.

[15] B. Denby and D. Schofield. Inclusion of risk assessment in open pit design and scheduling. Transactions of the Inst. Min. Metall., Section A: Min. industry, 104:A67-A71, January April 19951995

[16] B. Denby and D. Schofield. The use of genetic algorithms in underground mine scheduling. In Proceedings of the 25th International Symposium Application of Computers Mineral Industries, pages 389-394, Brisbane, Australia, 1995.
[17] B. Denby, D. Schofield, and T. Surme. Genetic algorithms for flexible scheduling of open pit operations. In Proceedings of APCOM 27th International Symposium Mathematics in the Mineral Industries, London, UK, 1998.

[18] G. C. Goodwin, M. M. Seron, and J. A. De Doná. Constrained Control and Estimation: An Optimisation Approach. Springer, New-York, 2005.

[19] J. M. Maciejowski. Predictive Control with Constraints. Prentice Hall, 2002.

[20] S. J. Qin and T. A. Badgwell. An overview of industrial model predictive control technology. In Chemical Process Control-V, CACHE, AIChE, pages 232-256, 1997.

[21] D. E. Quevedo and G. C. Goodwin. Moving horizon design of discrete coefficient FIR filters. IEEE Transactions on Signal Processing, 2004.

[22] A. Richards and J.P. How. Mixed-integer programming for control. In Proceedings of the 2005 American Control Conference, Portlan, Oregon, June 2005.

[23] J. A. Rossiter. Model-Based Predictive Control. CRC Press, 2003.

[24] Peter Stone, Gary Froyland, Merab Menabde, Brian Law, Reza Pasyar, and Peter Monkhouse. Blasor-blended ironore mine planning optimisation at Yandi. The Australasian Institute of Mining preprint, 2004.

[25] G. S. Thomas. Optimization and scheduling of open pits via genetic algorithms and simulated annealing. In Proceedings of the 1st International Symposium on Balkema Publisher, pages 44-59, Rotterdam, The Netherlands, 1996. 